\title{
Prevalence and antibiotic susceptibility of methicillin-resistant Staphylococcus aureus in the surgical ICU of General Hospital "Prim. dr Abdulah Nakaš" Sarajevo - our experience
}

\author{
Matković D. ${ }^{1}$, Muminagić-Hamza L. ${ }^{1}$, Štraus S. ${ }^{2}$
}

1 General Hospital 'Prim. Dr. Abdulah Nakaš' Sarajevo, Dept of Anaesthesiology \& Intensive Care, Sarajevo, Bosnia and Herzegovina, ${ }^{2}$ University Clinical Center Sarajevo, Institute for Heart Disease - Cardiosurgery Clinic, Dept of Intensive Care, Sarajevo, Bosnia and Herzegovina

BACKGROUND AND GOAL OF STUDY

The incidence of methicillin-resistant Staphylococcus aureus (MRSA) infections has declined over time in the USA, Europe and Canada, but increased in sub-Saharian Africa, India, LatinAmerica and Australia. It is not clear whether MRSA is replacing methicillin-susceptible S.aureus infections, or just adding to its overall incidence. With the varying regional patterns of resistance and the importance of those infections, it is prudent to know the local prevalence of MRSA and its' antibiotic sensitivity pattern. The goal of this study is to determine the prevalence and antibiotic susceptibility of health-care associated MRSA infections in the surgical ICU of the General Hospital "Prim. dr Abdulah Nakaš” Sarajevo.

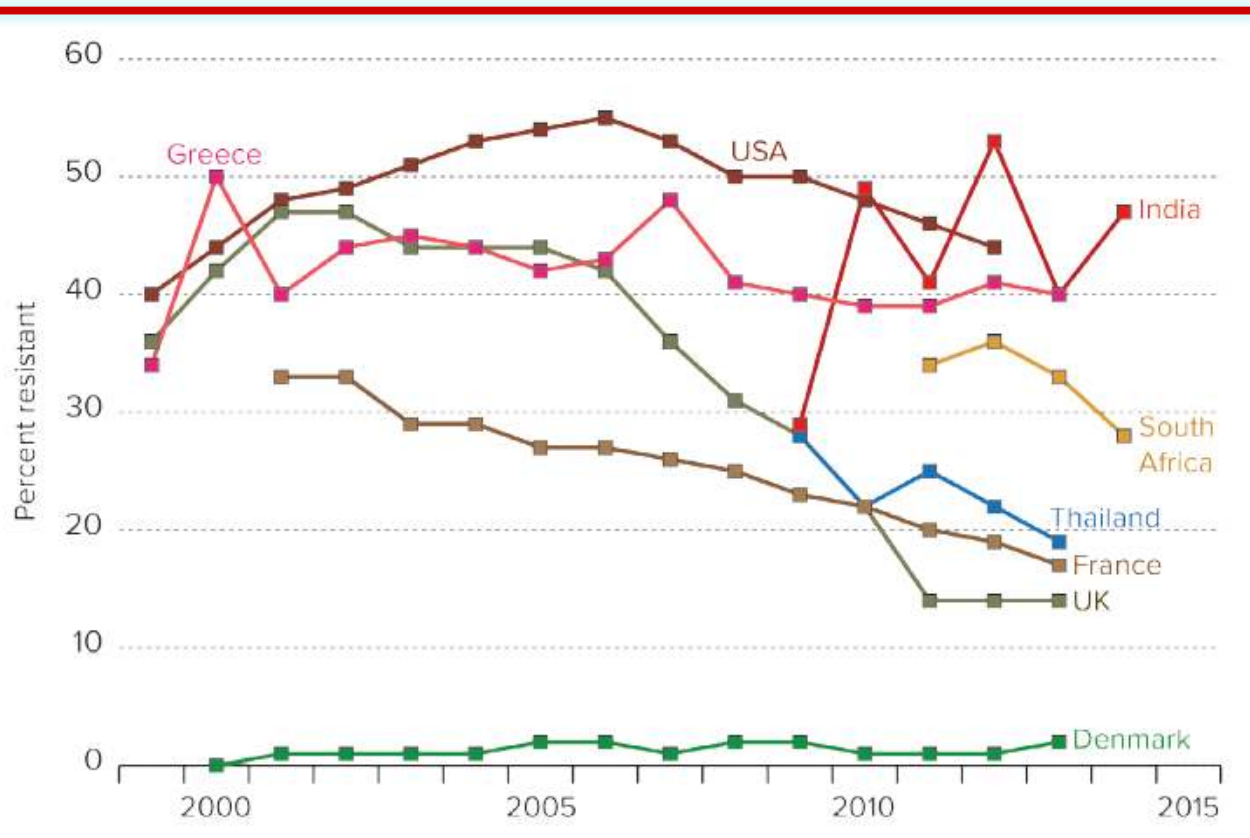

Fig. 1. Percentage of methicillin-resistant Staphylococcus aureus isolates in selected countries, 1999-2014 [1]

\section{MATERIALS AND METHODS}

This study is a retrospective data analysis of the results of microbiological testing of surgical ICU patients in the General Hospital "Prim. dr Abdulah Nakaš" Sarajevo, during the period 01/01/2014 to 10/12/2015. Identification of S.aureus was confirmed by standard methods, and antibiotic susceptibility testing was performed according to the Kirby-Bauer disc diffusion method on Müller-Hinton agar. Oxacillin, cloxacillin, penicillin and cefoxitin were used to test the resistance of isolated S.aureus strains to methicillin. Data were analyzed by methods of descriptive statistics.

\section{RESULTS AND DISCUSSION}

Out of the total of 96 nosocomial infections, in 16 cases (16.6\%) S.aureus was the identified causative agent, and in $8(50 \%)$ patients it was found to be a methicillin-resistant strain. Among them, 6 patients $(75 \%)$ were older than 70 years and 2 patients (25\%) 55-65 years. MRSA was isolated from sputum in 4 cases and from surgical wound swabs in 5 cases, with one patient having MRSA isolated from both samples. There were no proven MRSA bloodstream infections. Lethal outcome was observed in $3(37,5 \%)$ patients. Every isolated MRSA strain was sensitive to vancomycin, $75 \%$ to amikacin, $50 \%$ to amoxycillin+clavulonic acid and ampicillin+sulbactam and $25 \%$ to ciprofloxacin. All the MRSA isolates were resistant to 3 or more antibiotics tested.

\begin{tabular}{|lcc|}
\hline Specimen types & $\begin{array}{c}\text { Staphylococcus aureus } \\
\text { S.aureus }\end{array}$ & $\begin{array}{c}\text { Methicillin-resistant } \\
\text { S.aureus }\end{array}$ \\
\hline Sputum & 0 & 4 \\
\hline $\begin{array}{l}\text { Surgical wound } \\
\text { swab }\end{array}$ & 3 & 5 \\
\hline Blood & 3 & 0 \\
\hline $\begin{array}{l}\text { Central venous } \\
\text { catheter tip }\end{array}$ & 1 & 0 \\
\hline Urine & 1 & 0 \\
\hline
\end{tabular}

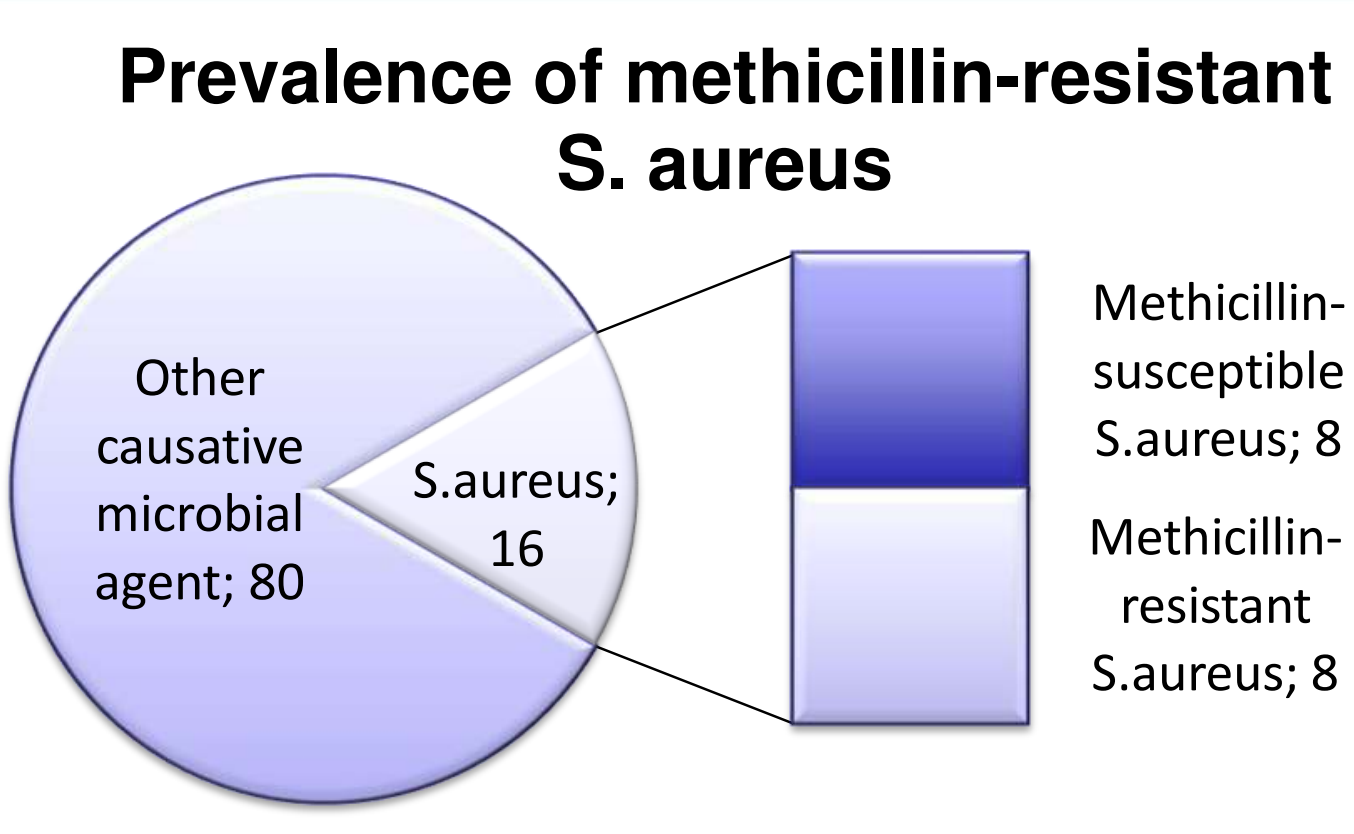

Methicillin-resistant S.aureus antibiotic sensitivity

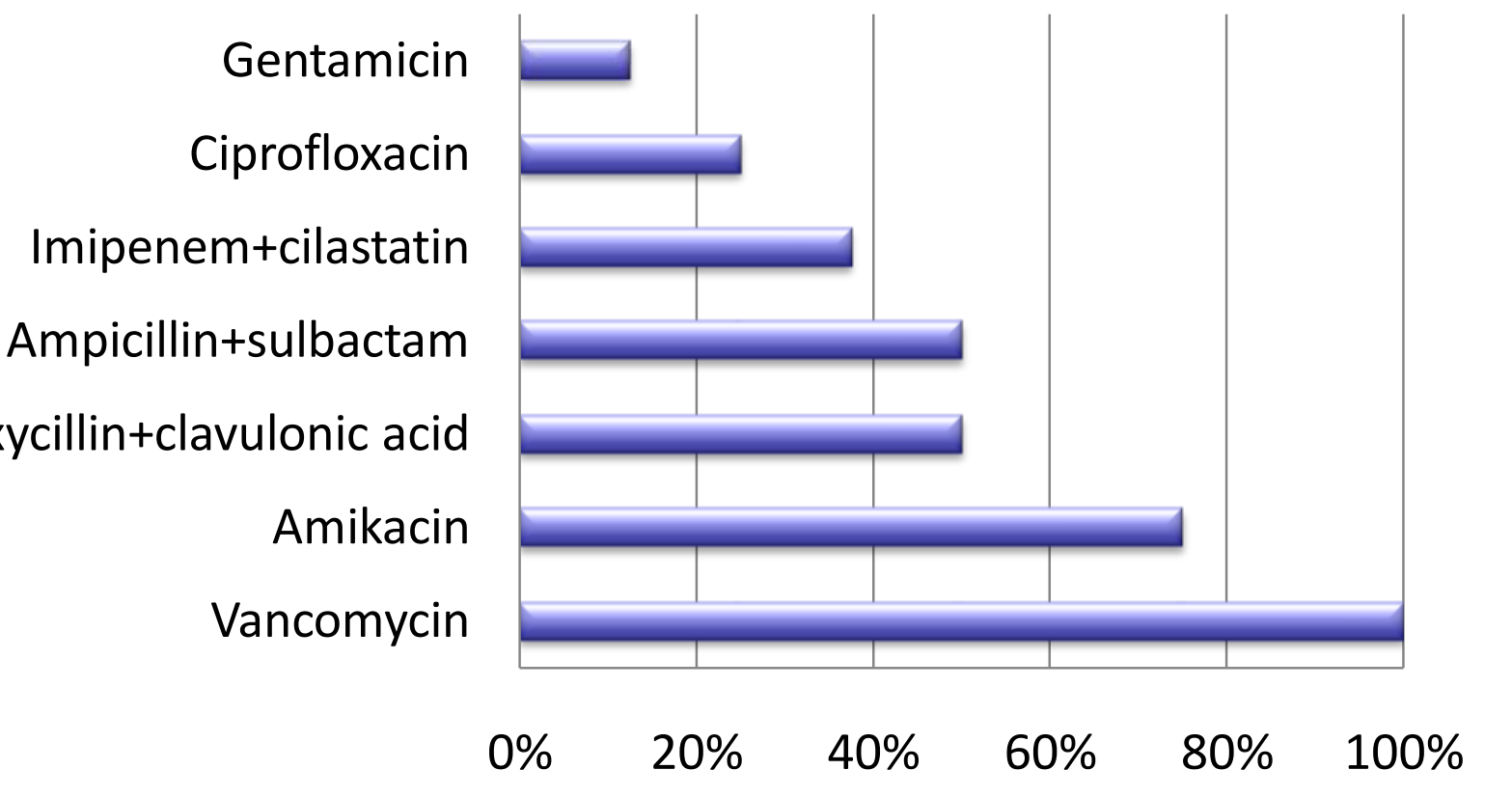

\section{CONCLUSION}

In our experience, MRSA infections account for half of the nosocomial S.aureus infections, with a $100 \%$ sensitivity of the isolated strains to vancomycin and a relative high sensitivity to amikacin.

[1]. Center for Disease Dynamics, Economics \& Policy. 2015, State of the World's Antibiotics, 2015. CDDEP: Washington, D.C.

[2]. Mostofsky E, Lipsitch M, Regev-Yochay G. Is methicillin-resistant Staphylococcus aureus replacing methicillin-susceptible

S.aureus? J Antimicrob Chemother 2011; 66: 2199-2114 